\title{
CHARACTERIZATION OF CERTAIN CLASSES OF FUNCTIONS
}

\section{GEN-ICHIRÔ SUNOUCHI}

(Received December 4, 1961)

1. Introduction. Let $f(x)$ be an integrable function, with period $2 \pi$ and let its Fourier series be

$$
f(x) \sim \sum_{k=0}^{\infty} A_{k}(x)=a_{0} / 2+\sum_{k=1}^{\infty}\left(a_{k} \cos k x+b_{k} \sin k x\right) .
$$

In the theory of the best approximation, we gave a class of functions $f(x)$ for which

$$
A_{0}(x)+\sum_{k=1}^{\infty} k^{\alpha} A_{k}(x) \in L^{\infty}(0,2 \pi)(0<\alpha<\infty) .
$$

This is the saturation class for uniform approximation of $f(x)$ by the Riesz typical means $\left(R, n^{\alpha}, 1\right)$ of its Fourier series, (Sunouchi-Watari [5]). This class of functions was already observed by Nagy [2] and Stečkin [4].

In the present note, we identify this class $(0<\alpha<1)$ with the class of functions $f(x)$ which satisfy

$$
\int_{u}^{\infty} \frac{f(x+t)+f(x-t)-2 f(x)}{t^{1+\alpha}} d t=O(1),
$$

uniformly in $u$.

At the beginning, we give a new general theorem of saturation from which the saturation theorem concerning $\left(R, n^{\alpha}, 1\right)$ means is derived. We show the above characterization and related theorems in the next paragraph. Incidently this consideration suggests definition of new summability methods of series. This is given in the last paragraph. These summability methods are generalization of $K$-method of Zygmund [7].

2. Theorems on saturation. Let

$$
g_{k}(n),(k=0,1,2, \ldots \ldots), g_{0}(n)=1
$$

be a summation matrix and denote a family of approximation of (1.1) by

$$
P_{n}(x, f)=\sum_{k=0}^{\infty} g_{k}(n) A_{k}(x)
$$

THEOREM 1. Suppose that 


$$
\lim _{n \rightarrow \infty} \frac{1-g_{k}(n)}{\varphi(n) \psi(k)}=c \neq 0(k=1,2, \ldots \ldots)
$$

where $\varphi(n)$ is positive non-increasing function and $\psi(k)$ is non-vanishing. Then for any continuous $f(x)$,

(I) $\left\|f(x)-P_{n}(x, f)\right\|_{c}=o\{\varphi(n)\}$, if and only if $f(x)=a$ constant.

(II) If $\left\|f(x)-P_{n}(x, f)\right\|_{c}=O\{\phi(n)\}$, then the trigonometric series

$$
f^{[\psi]}(x)=\sum_{k=1}^{\infty} \psi(k) A_{k}(x) \in L^{\infty}(0,2 \pi) .
$$

(III) If $f^{[\psi]}(x)$ belongs to class $L^{\infty}(0,2 \pi)$, and

$$
\lambda_{k}(n)=\frac{1-g_{k}(n)}{\phi(n) \psi(k)}
$$

is a $\left(L^{\infty}, L^{\infty}\right)$-multiplier uniformly in $n$, then

$$
\left\|f(x)-P_{n}(x, f)\right\|_{c}=O\{\phi(n)\} .^{1)}
$$

PROOF. Propositions (I) and (II) are proved in the previous paper (Sunouchi-Watari [5]). Proposition (III) is essentially given in the paper of Butzer [1], concerning Fourier integral. We give here a simple proof.

$f(x)-P_{n}(x, f)$ is continuous and

$$
\begin{gathered}
f(x)-P_{n}(x, f) \sim \sum_{k=1}^{\infty}\left\{1-g_{k}(n)\right\} A_{k}(x), \\
\left\{f(x)-P_{n}(x, f)\right\} / \varphi(n) \sim \sum_{k=1}^{\infty} \lambda_{k}(n) \psi(k) A_{k}(x),
\end{gathered}
$$

where $\lambda_{k}(n)$ is defined by (2.4). From the hypotheses, $f^{[\psi]}(x) \in L^{\infty}(0,2 \pi)$ and $\lambda_{k}(n)$ is $\left(L^{\infty}, L^{\infty}\right)$-multiplier uniform in $n$. Hence we have

$$
\left\|f(x)-P_{n}(x, f)\right\|_{\infty}=O\{\phi(n)\} .
$$

However, $f(x)-P_{n}(x, f)$ is continuous and

$$
\left\|f(x)-P_{n}(x, f)\right\|_{c}=O\{\phi(n)\} .
$$

Thus we show the proposition III.

REMARK 1 . If we take $L$-norm for approximation instead of $C$-norm, we get a variant of Theorem 1 .

1) A.H. Turetskii [On classes of saturation in the space $C$, Izvestja Akad. Nauk 25(1961), 411-442 (Russian)], claimed that if $\left\|P_{n}(x, f)\right\|_{\infty} \leqq M\|f\|_{\infty}$ and $f[\psi] \in L^{\infty}$, where $\psi(k)=k^{p}$ ( $p$ is a positive integer), then $\left\|f(x)-P_{r_{s}}(x, f)\right\|_{c}=\mathrm{O}\{p(n)\}$. But the present author cannot follow his proof. The present author thinks that this proposition would be false without any additional hypothesis. 
THEOREM 1'. If $f(x) \in L^{1}(0,2 \pi)$ and the condition (2.3) is satisfied then

(I') $\left\|f(x)-P_{n}(x, f)\right\|_{1}=o\{\varphi(n)\}$, if and only if $f(x)=a$ constant.

(II') If $\left\|f(x)-P_{n}(x, f)\right\|_{1}=O\{\phi(n)\}$, then the trigonometric series $f^{[\psi]}(x)$ belongs to class $d V(0,2 \pi)$, that is, $f^{[\psi]}(x)$ is Fourier-Stieltjes series of a function of bounded variation.

(III') If $f^{[\psi]}(x)$ belongs to class $d V(0,2 \pi)$ and $\lambda_{k}(n)$ is a $(d V, d V)$-multiplier uniform in $n$, then

$$
\left\|f(x)-P_{n}(x, f)\right\|_{1}=O\{\phi(n)\} .
$$

Proof is done by the same method with that of Theorem 1, so we omit it.

Since we know that $\left(L^{\infty}, L^{\infty}\right)=(d V, d V)$, the second condition of (III) is the same with that of (III). If we take $L^{p}(1<p<\infty)$ norm, then this is replaced by the condition such as $\lambda_{k}(n)$ is a $\left(L^{p}, L^{p}\right)$-multiplier uniform in $n$. It is well known that a $\left(L^{\infty}, L^{\infty}\right)$-multiplier is a $\left(L^{p}, L^{p}\right)$-multiplier. Hence it is sufficient for us to prove that $\lambda_{k}(n)$ is a $\left(L^{\infty}, L^{\infty}\right)$-multiplier for all these norms.

We set, for $0<\alpha<\infty$,

$$
R_{n}^{\alpha}(x, f)=\sum_{k=0}^{n-1}\left(1-k^{\alpha} / n^{\alpha}\right) A_{k}(x) .
$$

THEOREM 2. (I) $\left\|f(x)-R_{n}^{\alpha}(x, f)\right\|_{c}=o\left(n^{-\alpha}\right)$, if and only if $f(x)=a$ constant.

(II) If $\left\|f(x)-R_{n}^{\alpha}(x, f)\right\|_{c}=O\left(n^{-\alpha}\right)$, then $\sum k^{\alpha} A_{k}(x)$ belongs to class $L^{\infty}(0,2 \pi)$.

(III) If $\sum k^{\alpha} A_{k}(x)$ belongs to class $L^{\infty}(0,2 \pi)$, then

$$
\left\|f(x)-R_{n}^{\alpha}(x, f)\right\|_{c}=O\left(n^{-\alpha}\right) .
$$

We proved this theorem already (Sunouchi-Watari [5]). But we give here a new proof in the view of Theorem 1 .

Since

$$
\varphi(n)=n^{-\alpha}, \psi(k)=k^{\alpha}
$$

and it is easily seen that $\lambda_{k}(n)$ is quasi-convex uniformly in $n$, that is

$$
\sum_{k=1}^{\infty} k\left|\lambda_{k-1}(n)-2 \lambda_{k}(n)+\lambda_{k+1}(n)\right|
$$

is uniformly bounded. Hence $\lambda_{k}(n)$ is a $\left(L^{\infty}, L^{\infty}\right)$-multiplier uniformly in $n$. Theorem 1 implies Theorem 2.

3. Characterization of certain classes of functions. In the preceeding 
paragraph, we derived a class of functions $f(x)$ for which $\sum k^{\alpha} A_{k}(x) \in L^{\infty}(0,2 \pi)$. We shall characterize this class by the term of $f(x)$.

THEOREM 3. $\sum k^{\alpha} A_{k}(x) \in L^{\infty}(0,2 \pi)(0<\alpha<1)$, if and only if,

$$
\int_{u}^{\infty} \frac{f(x+t)+f(x-t)-2 f(x)}{t^{1+\alpha}} d t=O(1)
$$

uniformly in $u$ and $x$.

PROOF. We set

$$
\begin{aligned}
\Delta^{2} f(x, t) & =f(x+t)+f(x-t)-2 f(x) \\
& \sim-4 \sum_{k=1}^{\infty} \sin ^{2}(k t / 2) A_{k}(x) .
\end{aligned}
$$

Multiplying by $t^{-(1+\alpha)}$ and integrating termwise in the interval $(u, \infty)$,

$$
\int_{u}^{\infty} \frac{\Delta^{2} f(x, t)}{t^{1+\alpha}} d \iota=-4 \sum_{k=1}^{\infty} A_{k}(x) \int_{u}^{\infty} \frac{\sin ^{2}(k t / 2)}{t^{1+\alpha}} d t .
$$

If (3.1) is valid, then for a fixed $u$, Fejér's mean of its Fourier series is bounded,

$$
\sum_{k=1}^{n-1}(1-k / n) A_{k}(x) \int_{u}^{\infty} \frac{\sin ^{2}(k t / 2)}{t^{1+\alpha}} d t=O(1) .
$$

However, this is true for every $u$ uniformly, and letting $u \rightarrow 0$ and observing

$$
\int_{0}^{\infty} \frac{\sin ^{2}(k t / 2)}{t^{1+\alpha}} d t=\frac{k^{\alpha}}{2^{\alpha}} \int_{0}^{\infty} \frac{\sin ^{2} x}{x^{1+\alpha}} d x=k^{\alpha} C_{\alpha},(0<\alpha<1)
$$

where $C_{\alpha}(\neq 0)$ is a constant depending only on $\alpha$, we get

$$
\sum_{k=1}^{n-1}(1-k / n) k^{\alpha} A_{k}(x)=O(1)
$$

This implies

$$
\sum_{k=1}^{\infty} k^{\alpha} A_{k}(x) \in L^{\infty}(0,2 \pi)
$$

Conversely we suppose that (3.3) is satisfied. Then from Theorem 2,

$$
\left\|f(x)-\sum_{k=1}^{n}\left(1-k^{\alpha} / n^{\alpha}\right) A_{k}(x)\right\|_{c}=O\left(n^{-\alpha}\right) .
$$

By Bernstein's theorem, $f(x) \in L i p \alpha$ and, by a theorem of Salem-Zygmund [3], 


$$
\begin{gathered}
-\frac{1}{\pi} \sin \frac{\pi \alpha}{2} \Gamma(\alpha+1) \int_{1-r}^{\infty} \frac{\Delta^{2} f(x, t)}{t^{1+\alpha}} d t \\
-\sum_{k=1}^{\infty} k^{\alpha} A_{k}(x) r^{k}=O(1),
\end{gathered}
$$

uniformly in $r$. Since, from (3.3),

$$
\sum_{k=1}^{\infty} k^{\alpha} A_{k}(x) r^{k}=O(1)
$$

we get

$$
\int_{u}^{\infty} \frac{\Delta^{2} f(x, t)}{t^{1+\alpha}} d t=O(1)
$$

uniformly. Thus the theorem is proved.

REMARK 2. Theorem 2 is true for $\alpha=1$. This is given by Zamansky [6]. Also Theorem 2 may be true for $1<\alpha<2$. Moreover the results may be extended to greater $\alpha$ with higher differences $\Delta^{k} f(x, t)$.

A counterpart of this theorem is the following

THEOREM 4. $\sum k^{\alpha} B_{k}(x) \in L^{\infty}(0,2 \pi)(0<\alpha<1)$, if and only if

$$
\int_{u}^{\infty} \frac{f(x+t)-f(x-t)}{t^{1+\alpha}} d t=O(1)
$$

uniformly in $u$ and $x$.

ProOF. We put

$$
\begin{aligned}
\Delta f(x, t) & =f(x+t)-f(x-t) \\
& \sim-2 \sum_{k=1}^{\infty} \sin k t B_{k}(x)
\end{aligned}
$$

where

$$
B_{k}(x)=b_{k} \cos k x-a_{k} \sin k x .
$$

On the same reason of Theorem 3,

$$
\begin{aligned}
& -\frac{1}{\pi} \int_{u}^{\infty} \frac{\Delta f(x, t)}{t^{1+\infty}} d t \\
& \quad=\frac{2}{\pi} \sum_{k=1}^{\infty} B_{k}(x) \int_{u}^{\infty} \frac{\sin k t}{t^{1+\infty}} d t .
\end{aligned}
$$

If (3.5) is bounded uniformly, then 


$$
\sum_{k=1}^{n-1}(1-k / n) B_{k}(x) \int_{u}^{\infty} \frac{\sin k t}{t^{1+\infty}} d t=O(1) .
$$

Letting $u \rightarrow 0$ and observing that

$$
\int_{0}^{\infty} \frac{\sin k t}{t^{1+\alpha}} t=k^{\alpha} \int_{0}^{\infty} \frac{\sin x}{x^{1+\alpha}} d x=k^{\alpha} D_{\alpha}
$$

where $D_{\alpha}$ is a constant, we have

$$
\sum_{k=1}^{n-1}(1-k / n) k^{\alpha} B_{k}(x)=O(1)
$$

This implies

$$
\sum_{k=1}^{\infty} k^{\alpha} B_{k}(x) \in L^{\infty}(0,2 \pi)
$$

On the contrary, we suppose (3.7) is satisfied, then, by Theorem 2,

$$
\left\|\tilde{f}(x)-\sum_{k=1}^{n}\left(1-k^{\alpha} / n^{\alpha}\right) B_{k}(x)\right\|_{c}=O\left(n^{-\alpha}\right) .
$$

Hence $\widetilde{f}(x) \in \operatorname{Lip} \alpha, f(x) \in \operatorname{Lip} \alpha$ and by a theorem of Salem-Zygmund [3], we have

$$
\begin{gathered}
-\frac{1}{\pi} \Gamma(\alpha+1) \cos \frac{\pi \alpha}{2} \int_{1-r}^{\infty} \frac{\Delta f(x, t)}{t^{1+\alpha}} d t \\
-\sum_{k=1}^{\infty} k^{\alpha} B_{k}(x) r^{k}=O(1) .
\end{gathered}
$$

However (3.7) implies

$$
\sum_{k=1}^{\infty} k^{\alpha} B_{k}(x) r^{k}=O(1)
$$

Therefore we have

$$
\int_{u}^{\infty} \frac{\Delta f(x, t)}{t^{1+\alpha}} d t=O(1)
$$

REMARK 3. Similarly we may consider another norms such as $L_{p}$-norm $(p \geqq 1)$.

4. New methods of summability. Above consideration suggests definition of new methods of summability. For a given series $\sum u_{k}$, we say that the series is summable to $s$ by the method $(\Re, \alpha)$, if 


$$
u_{0}+D_{\alpha} \sum_{k=1}^{\infty} u_{k} \int_{u}^{\infty} \frac{\sin k t}{k^{\alpha} t^{1+\alpha}} d t,\left(D_{\alpha}=\int_{0}^{\infty} \frac{\sin x}{x^{1+\alpha}} d x\right)
$$

converges for $u$ positive and small, and the limit of (4.1) for $u \rightarrow+0$ exists and equals $s$. And another method $\left(\Re^{*}, \alpha\right)$ is defined, if

$$
u_{0}+C_{\alpha} \sum_{k=1}^{\infty} u_{k} \int_{u}^{\infty} \frac{\sin ^{2}(k t / 2)}{k^{\alpha} t^{1+\alpha}} d t,\left(C_{\alpha}=\frac{1}{2^{\alpha}} \int_{0}^{\infty} \frac{\sin ^{2} x}{t^{1+\alpha}} d x\right)
$$

converges for $u$ positive and small, and the limit of (4.4) as $u \rightarrow+0$ exists and equals $s$. Then we shall show that these methods are regular.

THEOREM 5. Every series convergent to $s$, is summable by the method $(\Re, \alpha)$ or $\left(\Re^{*}, \alpha\right)$ to the same sum $s$.

PROOF. Convergency of (4.1) and (4.2) is evident. It is enough to verify the three conditions of regularity. The only condition which is not evident is that the sum of the absolute values of differences of the elements in each row remains bounded as $\alpha \rightarrow+0$. So we have to prove

$$
\sum_{k=1}^{\infty}\left|\int_{u}^{\infty} \Delta_{k}(t) d t\right|=O(1)
$$

where

$$
\Delta_{k}(t)=\frac{\sin (k-1) t}{t^{1+\alpha}(k-1)^{\alpha}}-\frac{\sin k t}{t^{1+\alpha} k^{\alpha}}
$$

or

$$
\Delta_{k}^{*}(t)=\frac{\sin ^{2}((k-1) t / 2)}{t^{1+\alpha}(k-1)^{\alpha}}-\frac{\sin ^{2}(k t / 2)}{t^{1+\alpha} k^{\alpha}}
$$

In case (4.3),

$$
\begin{aligned}
\int_{u}^{\infty} \Delta_{k}(t) d t & =\int_{(k-1) u}^{\infty} \frac{\sin x}{x^{1+\alpha}} d x-\int_{k u}^{\infty} \frac{\sin x}{x^{1+\alpha}} d x \\
& =\int_{(k-1) u}^{k u} \frac{\sin x}{x^{1+\alpha}} d x,
\end{aligned}
$$

and so

$$
\begin{aligned}
\left|\int_{u}^{\infty} \Delta_{k}(t) d t\right| & \leqq \int_{(k-1) u}^{u k}\left|\frac{\sin x}{x^{1+\alpha}}\right| d x \leqq \int_{(k-1) u}^{k u} x^{-\alpha} d x \\
& \leqq C u^{1-\alpha} k^{-\alpha}, \\
\left|\int_{u}^{\infty} \Delta_{k}(t) d t\right| & \leqq \mid \int_{(k-1) u}^{k u} \frac{|\sin x|}{x^{1+\alpha}} d x \leqq \frac{1}{(k u)^{1+\alpha}} \int_{(k-1) u}^{k u} d x
\end{aligned}
$$




$$
\leqq C k^{-(1+\alpha)} u^{-\alpha} \text {. }
$$

Using these estimations, we see that

$$
\begin{aligned}
& \sum_{k=1}^{\infty}\left|\int_{u}^{\infty} \Delta_{k}(t) d t\right|=\sum_{k \leqq u-1}+\sum_{k>u-1}=\sum_{1}+\sum_{2}, \\
& \sum_{1} \leqq C \sum_{k \leqq u-1} u^{1-\alpha} k^{-\alpha}=C u^{1-\alpha} \sum_{k \leqq u-1} k^{-\alpha}=O(1), \\
& \sum_{2} \leqq C \sum_{k>u-1} k^{-(1+\alpha)} u^{-\alpha}=C u^{-\alpha} \sum_{k>u-1} k^{-(1+\alpha)}=O(1) .
\end{aligned}
$$

Thus we get the required result.

On the other case (4.4), we may prove the same way. In this case

$$
\int_{u}^{\infty} \Delta_{k}^{*}(t) d t=\frac{1}{2^{\alpha}} \int_{(k-1)}^{k u} \frac{\sin ^{2} x}{x^{1+\alpha}} d x
$$

However we get

$$
\begin{aligned}
& \left|\int_{(k-1) u}^{k u} \frac{\sin ^{2} x}{x^{1+\alpha}} d x\right| \leqq \int_{(k-1) u}^{k u} x^{-\alpha} d x \leqq C u^{1-\alpha} k^{-\alpha} \\
& \left|\int_{(k-1) u}^{k u} \frac{\sin ^{2} x}{x^{1+\alpha}} d x\right| \leqq \frac{1}{(k u)^{1+\alpha}} \int_{(k-1) u}^{k u} d u \leqq C k^{-(1+\alpha)} u^{-\alpha} .
\end{aligned}
$$

Thus we have the same estimation of the integral, and we can complete the proof.

\section{REFERENCES.}

[1] P.L. BUTzER, Fourier-transform methods in the theory of approximation, Archive for Ratıonal Mechanics and Analysis, 5(1960), 390-415.

[2] B.S. NAGY, Sur une classe générale de procédés de sommation pour les séries de Fourier, Hungaria Acta Math., 1(1948), 1-39.

[3] R.SAlem AND A.ZYGMUND, Capacity of sets and Fourier series, Transaction Amer. Math. Soc., 59(1946), 23-41.

[4] S. B.STEČKIN, On the best approximation of some classes of functions by trigonometric polynomials, Izvestja Akad. Nauk., 20(1956), 643-648 (Russian).

[5] G.SUNOUCHI AND C. WATARI, On determination of the class of saturation in the theory of approximation of functions II, Tôhoku Math. Journ., 11(1959), 480-488.

[6] M.ZAMANSKY, Classes de saturation de certaines procédes d'approximation des series de Fourier des fonctions continues, Ann. Sci. Ecole Normale Sup., 66(1949), 19-93.

[7] A.ZYGMUND, On certain methods of summability associated with conjugate trigonometric series, Studia Math., 10(1948), 97-105.

MATHEMATICAl. INSTITUTE, TÔHOKU UNIVERSity 